\title{
Controlling hypertension immediately post stroke: a cost utility analysis of a pilot randomised controlled trial
}

\author{
Edward CF Wilson ${ }^{1 *}$, Gary A Ford ${ }^{2}$, Tom Robinson ${ }^{3}$, Amit Mistri ${ }^{3}$, Carol Jagger ${ }^{4}$, John F Potter ${ }^{1}$
}

\begin{abstract}
Background: Elevated blood pressure (BP) levels are common following acute stroke. However, there is considerable uncertainty if and when antihypertensive therapy should be initiated.

Method: Economic evaluation alongside a double-blind randomised placebo-controlled trial (National Research Register Trial Number N0484128008) of 112 hypertensive patients receiving an antihypertensive regimen (labetalol or lisinopril) within 36 hours post stroke versus 59 receiving placebo. Outcomes were incremental cost per incremental: QALY, survivor, and patient free from death or severe disability (modified Rankin scale score $<4$ ) at three months and 14 days post stroke.

Results: Actively treated patients on average had superior outcomes and lower costs than controls at three months. From the perspective of the acute hospital setting, there was a $96.5 \%$ probability that the incremental cost per QALY gained at three months is below $£ 30,000$, although the probability may be overstated due to data limitations.

Conclusion: Antihypertensive therapy when indicated immediately post stroke may be cost-effective compared with placebo from the acute hospital perspective. Further research is required to confirm both efficacy and costeffectiveness and establish whether benefits are maintained over a longer time horizon.
\end{abstract}

\section{Background}

Approximately 52,000 patients experience first stroke [1], and 135,000 experience first or recurrent stroke in England and Wales each year [2]. It is the third biggest cause of death and the most important single cause of severe adult disability [3]. The societal cost of stroke to England and Wales is estimated at $£ 7 \mathrm{bn}$, of which $40 \%$ are direct care costs, $35 \%$ informal care, and the remaining $25 \%$ indirect costs (lost productivity) [4].

Elevated blood pressure (BP) levels are common following onset of acute stroke, and observational data suggest that both high and low BP levels are associated with poor short and long term prognosis [5-16]. The acute management of post-stroke BP changes is a matter of some debate, with considerable differences of opinion on when to initiate antihypertensive therapy [17]. A Cochrane review of BP manipulation following stroke

\footnotetext{
* Correspondence: ed.wilson@uea.ac.uk

${ }^{1}$ Faculty of Health, University of East Anglia, Norwich, UK
}

concluded that there was insufficient evidence to evaluate the effect of changes on patient outcomes [18].

In view of the uncertainty surrounding appropriate response to $\mathrm{BP}$ control in the acute post-stroke phase, the Control of Hypertension and Hypotension Immediately Post Stroke (CHHIPS) trial (National Research Register Trial Number N0484128008) aimed to establish the safety, efficacy and cost-effectiveness of reducing BP with labetalol or lisinopril in hypertensive patients with acute cerebral infarction or haemorrhage, and of raising $\mathrm{BP}$ with phenylephrine in hypotensive patients with ischaemic stroke.

As resources are finite, decision making requires consideration not only of the benefits to a patient of a health care intervention, but its impact on other patients consuming other diverse health care services: committing resources to one intervention means they cannot be employed, or must be withdrawn from, elsewhere. An economic evaluation considers the cost and consequences of two or more treatment strategies, and shows 
the change in both cost and outcome by adopting a new strategy in place of old [19]. The change in cost divided by the change in outcome (the incremental cost-effectiveness ratio or ICER) is then compared with a maximum 'threshold'. This threshold can be interpreted as the cost-effectiveness of the least efficient service currently provided by the health service (although alternative interpretations of the threshold exist). If the ICER is below this threshold, adopting the new treatment (and by implication ceasing the least efficient service) will improve the net health gain to the population. Conversely, adopting a treatment whose ICER is above the threshold will lead to a net reduction in health gain to the population. An outcome measure commonly used to make these comparisons is the Quality Adjusted Life Year (QALY), and the threshold in the UK is considered to be in the region of $£ 20,000-£ 30,000$ per QALY gained [20].

We report a cost-utility and cost-effectiveness analysis of therapeutically reducing blood pressure compared with no therapeutic reduction in blood pressure in hospitalised hypertensive patients with acute cerebral infarction or haemorrhage.

\section{Methods}

Full details of the methods and outcome measures in the study are reported elsewhere [21-23]. The study was designed to include both pressor and depressor trial arms. Due to low recruitment, the pressor arm of the trial was terminated early. We therefore report costs and outcomes relating to the depressor arm only.

Briefly, 179 patients aged $18+$ years with a clinical diagnosis of stroke (cerebral infarct or haemorrhage) with onset $\leq 36$ hours and systolic blood pressure (SBP) $\geq 160 \mathrm{mmHg}$ were enrolled into this randomised double-blind placebo-controlled trial. Exclusion criteria included on antihypertensive therapy at time of stroke onset (amended during study to allow inclusion of dysphagic patients on antihypertensive therapy) or an urgent indication for BP lowering, significant co-morbidity, or a life expectancy $\leq$ six months due to nonstroke causes prior to stroke onset.

Following baseline assessment (SBP levels, time of stroke onset, swallowing status, functional assessments including modified Rankin scale (mRS) and National Institute of Health Stroke Scale (NIHSS)), patients were randomised on a 2:1 ratio between active treatment and placebo.

Active treatment comprised stepped doses of oral (for non-dysphagic) or intravenous/sublingual routes of labetalol or lisinopril respectively with a target SBP of 145-155 $\mathrm{mmHg}$ or a SBP fall of $\geq 15 \mathrm{mmHg}$. Additional doses were administered at 4 and 8 hours post randomisation if targets were not met. Controls were administered matching placebo, and the regimen continued for 14 days post randomisation. Dysphagic patients underwent similar titrated dosing but with sublingual lisinopril $5 \mathrm{mg}$, intravenous labetalol $50 \mathrm{mg}$ or matching placebo for 72 hours, then oral therapy (if possible), or via nasogastric tube until day 14. Subsequently all patients followed local guidelines as regards antihypertensive therapy (usually an ACE inhibitor and/or diuretic). At day 14 and 3 months post randomisation, mRS was completed.

Baseline and two week assessments were performed by research staff at the local centres. Three month followup was by telephone administered from the trial coordinating centre. Where participants were not able to recall date of discharge at the three month follow-up, the local research staff were contacted to obtain the date from hospital records.

The primary outcomes were incremental cost per incremental survivor and incremental cost per incremental QALY gained at 3 months post randomisation with active treatment versus placebo. Secondary analyses comprised incremental cost per incremental: patient with death or severe disability (defined as mRS score < 4 ) at 14 days and 3 months, and survivor and QALY gained at 14 days.

Utilities were mapped to mRS scores estimated from a study of 459 individuals eliciting utilities from mRS scores using the time trade-off (TTO) approach [24].

The analysis was conducted from the perspective of the acute hospital. Hence resource use data comprised patient length of stay and study drug consumption. The price year of the study was 2006. Length of stay (LoS) was calculated as the difference between date of death or discharge and date of randomisation. The bulk of hospitalisation costs tend to be skewed towards the first few days of admission and the National Schedule of Reference Costs 2006 [25] estimates the mean cost of a stroke admission at $£ 2642$, with a mean length of stay of 11 days, and a daily cost of excess bed-days of $£ 176$. We therefore approximated the cost of an admission as:

Cost of admission $=2642+(\operatorname{LoS}-11) * 176$

Per patient cost of study drugs was estimated as number of tablets or vials multiplied by unit cost (lisinopril @ $£ 1.34 / 285 \mathrm{mg}$ tabs, labetalol @ £3.79/56 $50 \mathrm{mg}$ tabs and $£ 2.12 / 20 \mathrm{ml}$ ampoule[26]). Placebo was costed at zero.

We present results as quantities of resource use and total cost, and outcomes by treatment group (active treatment vs placebo). The incremental cost-effectiveness ratio (ICER) was calculated as

$$
\mathrm{ICER}=(\mathrm{C} 2-\mathrm{C} 1) /(\mathrm{E} 2-\mathrm{E} 1)
$$

Uncertainty in the point estimate ICER was investigated by means of a non-parametric bootstrap with 
1000 replications. This was used to estimate confidence intervals around incremental cost and outcomes, and to generate the cost-effectiveness acceptability curve (CEAC). The CEAC shows the treatment (active or placebo) with the highest probability of being cost-effective at varying thresholds of willingness to pay for a unit of outcome, and is a means of expressing uncertainty around point estimates [27].

Results are presented as cost of each arm and increment, outcome from each arm and increment, and incremental cost-effectiveness (Table 1). The figures reported in Table 1 are based on complete case analysis (observations for which both cost and outcome data were available). Tables 2 and 3 report disaggregated resource use and cost, and outcomes using all observations for which cost or outcomes data were available (see Figure 1 for details).

\section{Results}

Of 179 patients randomised to the trial, eight were withdrawn post randomisation (see Potter et al. [23] for details of post-randomisation exclusions). Resource use data at 14 days and three months were available on 171 $($ Active $=112$, Placebo $=59)$ and $162($ Active $=105$, Placebo $=57)$ patients respectively. Utility data based on
mRS score at baseline and 14 days were available on all 171 patients. However at three months, mRS and hence mRS-based utilities and QALYs gained were available on 32 (Active $=18$, Placebo $=14$ ) patients. Survival status up to three months was recorded in all 171 patients. Therefore full cost and outcomes data were available on 171 (Active $=112$, Placebo $=59)$ patients at 14 days. At three months cost and survival data were available on 162 (Active $=105$, Placebo $=57$ ) patients, and cost and death/disability and cost and QALY data on 31 (Active = 17 , Placebo $=14)$ patients (Figure 1$)$.

There were no substantial differences in baseline characteristics between active and placebo treatment groups [23].

\section{Cost effectiveness}

There were no significant differences in cost or outcomes at 14 days (Table 1, analyses 1-3). At three months, active treatment per patient was (non-significantly) decreased by between $£ 1000$ and $£ 5511$ (Analyses 4-6 Table 1), with a gain of 0.044 QALYs (95\% CI $0.000,0.086$; Analysis 6 Table 1). Survival at three months favoured active treatment $(+11.5 \%$, 95\% CI: $+0.1 \%$, $+23.2 \%$; Analysis 4 Table 1$)$, as did proportion free from death or severe disability $(+34.0 \%$, 95\% CI: $+8.0 \%$, $+58.8 \%$; Analysis 5 Table 1$)$. The difference in

Table 1 Cost utility and cost effectiveness analyses at 14 days and 3 months (Complete case analysis)

\begin{tabular}{|c|c|c|c|c|c|c|c|c|c|c|}
\hline & \multicolumn{2}{|c|}{$\mathrm{n}$} & \multicolumn{2}{|r|}{$\underline{E}$} & \multicolumn{4}{|c|}{ Outcome } & \multirow[b]{2}{*}{ ICER } & \multirow[b]{2}{*}{$P(I C E R \leq £ 30 k)^{* *}$} \\
\hline & A & $\mathbf{P}$ & A & $\mathbf{P}$ & Increment $(95 \% \mathrm{Cl})$ & A & $\mathbf{P}$ & Increment $(95 \% \mathrm{Cl})$ & & \\
\hline 1. $14 d$ survival* & 112 & 59 & 2553 & 2525 & $28(-228,269) \S$ & 0.955 & 0.898 & $0.057(-0.028,0.144)$ & $£ 490$ & \\
\hline 2. 14d D\&D† & 112 & 59 & 2553 & 2525 & $28(-215,278) \S$ & 0.393 & 0.407 & $-0.014(-0.169,0.149)$ & [P dominant] & \\
\hline 3. $14 d$ CUA & 112 & 59 & 2553 & 2525 & $28(-226,268) \S$ & 0.028 & 0.027 & $0(-0.001,0.002)$ & $£ 76,162$ & $45.9 \%$ \\
\hline 4. $3 \mathrm{~m}$ survival* & 105 & 57 & 8234 & 9233 & $-1000(-3760,1588)$ & 0.905 & 0.789 & $0.115(0.001,0.232)$ & [A dominant] & \\
\hline $5.3 \mathrm{~m} \mathrm{D \& D}+$ & 17 & 14 & 5324 & 10835 & $-5511(-15183,1221)$ & 0.412 & 0.071 & $0.340(0.080,0.588)$ & [A dominant] & \\
\hline $6.3 \mathrm{~m} \mathrm{CUA \ddagger}$ & 17 & 14 & 5324 & 10835 & $-5511(-15712,1311)$ & 0.098 & 0.054 & $0.044(0.000,0.086)$ & [A dominant] & $96.5 \%$ \\
\hline
\end{tabular}

* Outcome = proportion surviving; + Outcome = proportion not dead or dependent (defined as mRS $<4$ ). $\neq$ Outcome $=\mathrm{QALYs}$ gained; $\S$ Differences in $95 \% \mathrm{C}$ around incremental cost in analyses $1,3 \& 5$ due to random error from non-parametric bootstrap.

** Threshold of $£ 30,000$ only appropriate to $£ / Q A L Y$.

Table 2 Mean Resource use and cost at 14 days and 3 months

\begin{tabular}{|c|c|c|c|c|c|c|c|c|c|c|}
\hline & \multicolumn{5}{|c|}{14 days } & \multicolumn{5}{|c|}{3 months } \\
\hline & \multicolumn{2}{|c|}{$\mathbf{N}$} & \multirow[b]{2}{*}{ A } & \multirow[b]{2}{*}{$\mathbf{P}$} & \multicolumn{3}{|c|}{$\mathbf{N}$} & \multirow[b]{2}{*}{ A } & \multirow[b]{2}{*}{$\mathbf{P}$} & \multirow[b]{2}{*}{ A-P } \\
\hline & A & $\mathbf{P}$ & & & A-P & A & $\mathbf{P}$ & & & \\
\hline Mean (SE) Los (days) & 112 & 59 & $11.49(0.402)$ & $11.36(0.577)$ & 0.14 & 105 & 57 & $43.77(3.38)$ & $49.47(7.28)$ & -5.7 \\
\hline Median (IQR) LoS (days) & 112 & 59 & $14(9,14)$ & $14(10,14)$ & 0 & 105 & 57 & $38(7,84)$ & $34(10,84)$ & 4.0 \\
\hline Patients still hospitalised n (\%) & 112 & 59 & $76(67.9)$ & $38(64.4)$ & $3.45 \%$ & 105 & 57 & $29(27.6)$ & $16(28.1)$ & $-0.45 \%$ \\
\hline Study drug consumption, vials. Mean (SE) & 112 & 59 & $4.7(0.7)$ & $5.7(1.1)$ & -1.02 & 112 & 59 & $4.7(0.7)$ & $5.7(1.1)$ & -1 \\
\hline Study drug consumption, tabs. Mean (SE) & 112 & 59 & $32.53(2.3)$ & $45.68(3.9)$ & -13.15 & 112 & 59 & $32.5(2.3)$ & $45.7(3.9)$ & -13.15 \\
\hline Cost of hospitalisation, $£$, mean (SE) & 112 & 59 & $2,548(71)$ & $2,525(101)$ & 23.78 & 105 & 57 & $8,230(594)$ & $9,233(1282)$ & $-1,003.60$ \\
\hline Cost of study drugs, $£$, mean (SE) & 112 & 59 & $4(1)$ & $0(0)$ & 4.14 & 105 & 59 & $4(1)$ & $0(0)$ & 4 \\
\hline Total cost, $£$, mean (SE) & 112 & 59 & $2,553(71)$ & $2,525(101)$ & $27.93(124)$ & 105 & 57 & $8,234(594)$ & $9,233(1282)$ & $-999.50(1413)$ \\
\hline
\end{tabular}

$\mathrm{SE}=$ Standard error of the mean, $\mathrm{IQR}=$ Inter-quartile range, $\mathrm{A}=$ active (labetalol or lisinopril), $\mathrm{P}=$ placebo. Note figures may vary

from those reported in Table 1 due to numbers of observations included (see Figure 1). 
Table 3 Outcomes at 14 days and 3 months

\begin{tabular}{cccccc}
\hline N & & & & & \\
\hline A & P & A & P & A-P & $\begin{array}{r}\text { P- } \\
\text { value }\end{array}$
\end{tabular}

\begin{tabular}{|c|c|c|c|c|c|c|}
\hline $\begin{array}{l}\text { Mean (SE) } \\
\text { utility }\end{array}$ & & & & & & \\
\hline Baseline & 112 & 59 & $0.892(0.007)$ & $0.899(0.008)$ & -0.007 & \\
\hline 14 days & 112 & 59 & $0.551(0.022)$ & $0.526(0.035)$ & 0.026 & 0.519 \\
\hline 3 months & 18 & 14 & $0.366(0.100)$ & $0.088(0.060)$ & 0.278 & 0.035 \\
\hline \multicolumn{7}{|c|}{ Mean (SE) QALYs gained } \\
\hline 14 days & 112 & 59 & $\begin{array}{r}0.028 \\
(0.0005)\end{array}$ & $\begin{array}{r}0.027 \\
(0.0007)\end{array}$ & 0.000 & 0.650 \\
\hline 3 months & 18 & 14 & $\begin{array}{r}0.102 \\
(0.0185)\end{array}$ & $\begin{array}{r}0.054 \\
(0.0116)\end{array}$ & 0.048 & 0.051 \\
\hline
\end{tabular}

\begin{tabular}{lrrrrrr}
\hline Survival $\mathrm{n}(\%)$ & & & & & \\
\hline 14 days & 112 & 59 & $107(95.54)$ & $53(89.83)$ & $5.71 \%$ & 0.148 \\
\hline 3 months & 112 & 59 & $102(91.07)$ & $47(79.66)$ & $11.41 \%$ & 0.034 \\
\hline mRS<4 $\mathrm{n}(\%)$ & & & & & & \\
\hline Baseline & 112 & 59 & $112(100)$ & $59(100)$ & $0.00 \%$ & \\
\hline 14 days & 112 & 59 & $44(39.29)$ & $24(40.68)$ & $-1.39 \%$ & 0.860 \\
\hline 3 months & 18 & 14 & $8(44.44)$ & $1(7.14)$ & $37.30 \%$ & 0.020
\end{tabular}

*Based on mapped mRS scores

**t-test for continuous variables, $\chi^{2}$ for proportions

$\mathrm{A}=$ active, $\mathrm{P}=$ placebo. Note figures vary from those reported in Table 1 due to numbers of observations included (see Figure 1).

the estimated cost increment between analysis 4 and analyses 5 and 6 is due to missing data: the figure quoted in analysis $4(£ 1000)$ is based on substantially more observations than that in analyses 5 and 6 (£5511), and is therefore subject to less sampling uncertainty.

At three months, therefore, according to all outcome measures, active treatment 'dominates' placebo (it is on average less expensive and more effective). We estimate a $96.5 \%$ probability of the incremental cost per QALY gained being below $£ 30,000$ (Table 1 Analysis 6), indeed irrespective of the threshold, the probability that treatment is cost-effective never falls below 92\%.

The above figures are based on complete case analysis. That is, observations were included in analyses 1-6 only where complete cost and outcome data were available (see Figure 1). We had complete survival data on all 171 patients at three months. However, we were only able to measure $\mathrm{mRS}$ and hence QALYs gained on 32 patients at 3 months. Therefore the estimate of incremental cost reported above does not include all observations for which cost data were available. Looking just at resource use data (and hence based on $\mathrm{n}=105$ active +57 placebo), we estimate an incremental cost at 3 months of -£1000 (95\% CI: -3450, 1451; Table 2). Similarly, we estimate incremental QALYs at 3 months at +0.048 (-0.0002, 0.0956; Table 3).

\section{Discussion}

To our knowledge, this is the first study examining the cost-effectiveness of antihypertensive medication immediately post stroke. Other studies have been in the context of primary or secondary prevention of cardio- or cerebrovascular events in hypertensive patients. These studies largely favour the use of preventative pharmacotherapy [28-30].

On average over three months, we found active treatment within the first 2 weeks of stroke onset to be both cost saving and outcome improving, leading to active treatment dominating placebo. However there are important caveats to bear in mind in interpreting the results. It should be noted that $95 \%$ confidence intervals around increments were of borderline statistical significance (e.g. Table 1, outcomes analyses 4, 5 and 6). It is highly likely that the analyses with small sample sizes (e.g. 5 and 6) are subject to selection bias due to potential correlation between health status and probability of providing outcomes data at three months (this is likely 'U-shaped': sicker individuals are less likely to respond to request for longer term follow-up data, whilst death is relatively easy to establish. Indeed, we had $\mathrm{mRS}$ and QALY data on $23(11,12)$ of 31 patients by virtue of knowledge of date of death).

This was a trial for which data collection proved to be problematic, particularly in terms of disability status at three month follow-up. The primary objective of the study was to assess whether disability and death at two weeks post stroke was affected by drug induced reduction of BP [23]. Study recruitment was only $11 \%$ of that for which it was powered, for a variety of reasons including the inherent difficulty in recruiting patients within the allowed time frame post ictus, and higher than anticipated prevalence of pre-treated hypertension (one of the exclusion criteria).

The economic evaluation component of this study was added following commencement of the trial via a protocol amendment, with research resources permitting only limited data collection. Therefore the analysis relied almost exclusively on patient-reported length of stay to determine the cost of active and placebo treatments (the cost of the study drugs was trivial), and the perspective of the analysis was thus restricted to the acute hospital admitting the stroke patient.

The use of self-reported length of stay is a common method for data collection in economic evaluations alongside trials. However, this is subject to recall bias. Studies of the reliability of self-reported data have reported mixed results $[31,32]$. The impact of this on the study depends on whether the average errors in length of stay are equal between the arms. Randomisation should ensure an even distribution of patients more 


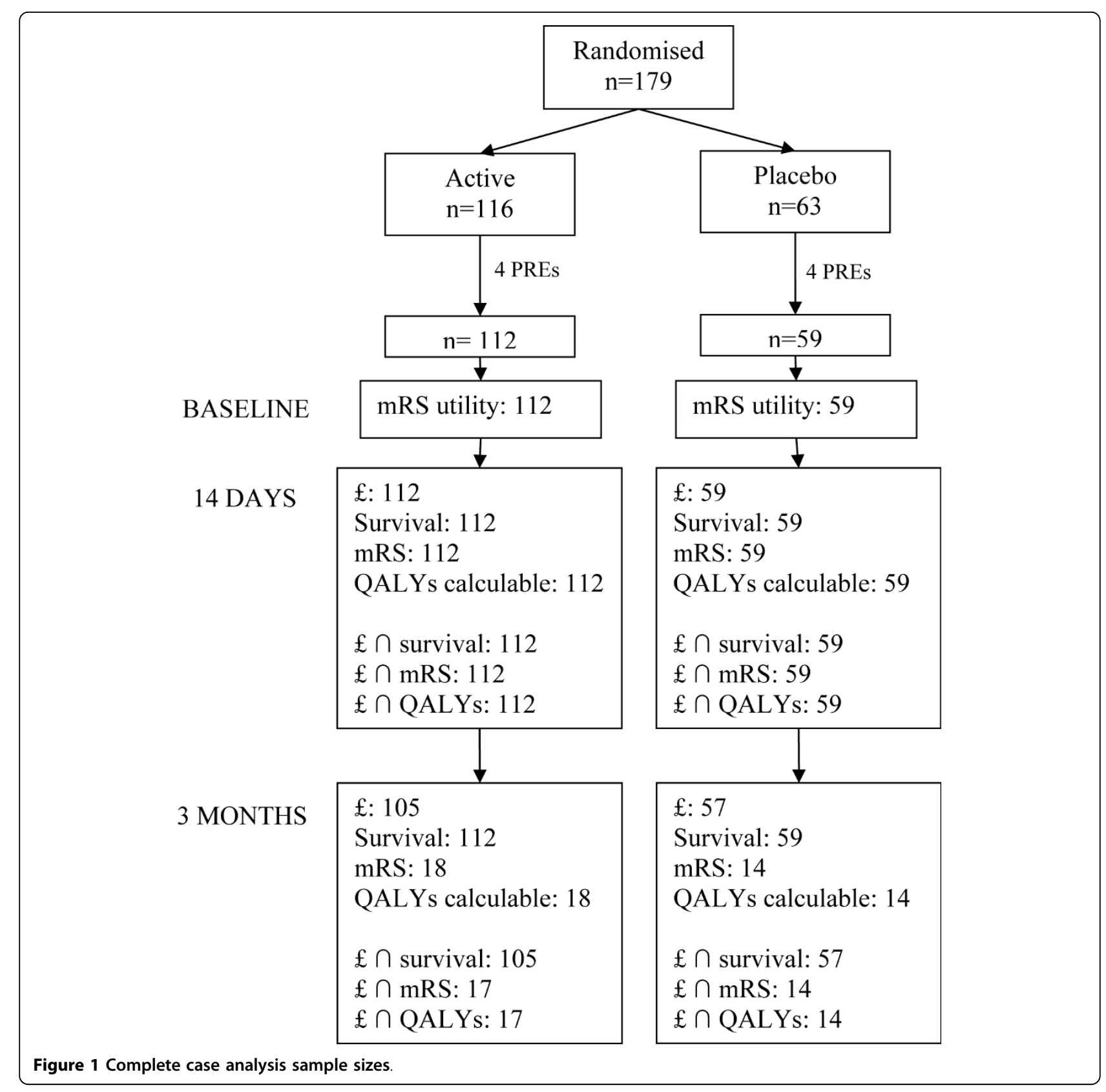

or less likely to misreport their length of stay ceteris paribus, but it is likely the error will increase with increasing length of stay. In common with all studies collecting resource use data in this way, this must be borne in mind in interpreting the results.

Costing based on length of stay with drug costs added to this may risk double counting if the unit cost used factors in an allowance for drugs. This is an issue common to many economic evaluations, and care must be taken to be sure of what is included in 'per episode' unit costs. In the context of this study, as drug costs were such a trivial component, the impact on the results would be negligible.

We did not document readmissions within this study. However, for this to affect the conclusion of the study, we estimate that patients in the treatment arm would on average, need 2.3 to 2.5 additional readmissions per patient over the three months compared with placebo. We consider such a large difference to be unlikely, indeed a priori it may be expected for there to be fewer readmissions in the active treatment arm. (Please see Appendix 1 for details). 
The EQ-5D generic quality of life instrument was included within this study by protocol amendment. As this was after baseline measurements had been taken, and due to the small numbers of observations, it was decided to map the mRS scores to utilities and hence QALYs gained, rather than use the EQ-5D data [23]. The analysis did not take into account uncertainty in the TTO valuations of the MRS scale [24]. Therefore we may have underestimated the decision uncertainty, although this would not affect the point estimate results.

We only had relatively small numbers of observations for analyses 5 and 6 (reporting incremental cost per incremental death and disability avoided and QALY; Table 1). There is therefore danger of the groups being unbalanced. A comparison of baseline characteristics of patients included in these analyses shows that they remain broadly balanced (the tables in additional files 1 and 2 show the baseline characteristics of patients included in analysis 4 and analyses 5 \& 6 respectively), and results of these analyses are consistent with those of analysis 4, based on a much larger patient sample.

Given the limitations outlined above, the question that must be asked is whether any conclusions can be drawn from such data about a) cost-effectiveness from the acute setting perspective, and b) the generalisability of this restricted analytic perspective to wider societal costeffectiveness over a longer horizon. Length of stay has been shown to be the major determinant of acute care cost $[33,34]$ and therefore our cost estimates could be plausible indicators of the incremental cost of treating patients under active or placebo treatment in the acute setting. The issue of generalisability to wider perspectives is of particular relevance given the high care needs and associated cost of many stroke survivors (both in terms of health and social services, and informal carer time $[4,35])$.

This can only by answered either through long-term prospective studies, or through decision analytic modelling. Such a prospective study may be prohibitively expensive and time consuming to conduct. The modelling approach is therefore recommended as a means of generating an answer within a reasonable time frame, and the results of this study should be seen as a valuable input into such an exercise, rather than a definitive estimate of the cost-effectiveness of antihypertensive medication immediately post stroke. Once such a model has been developed, value of information analysis may be used to estimate the likely return from a larger scale (and longer term) trial [36].

Future trials of treatments in this area wishing to incorporate an economic aspect to their investigations should include a) generic quality of life measurement alongside any disease specific or clinical endpoints and b) resource use data collection from the outset.
Consideration should be given as to whether at the very least quality of life and place of residence (i.e. own home, care home, nursing home) could be relatively easily measured at, say, six months and one year post intervention to lengthen the time horizon of any such study at minimal additional research cost.

\section{Conclusion}

Antihypertensive therapy in hypertensive patients immediately post stroke may be effective and cost-effective compared with placebo from the acute hospital perspective at three months post ictus. Further research, in particular decision analytic modelling, is required to confirm both efficacy and cost-effectiveness and whether benefits are maintained over a longer time horizon. The data from this study form a useful input into such a model.

\section{Appendix 1: The estimated impact of excluding readmissions}

- At three months, point estimate results were that intervention was $£ 5,324$ less expensive than control, and resulted in 0.044 more QALYs, yielding an ICER of $-£ 121,000$ (intervention dominant).

- For the ICER to be below $£ 20,000$, the cost in the intervention arm could rise by $£ 6204$ (yielding an incremental cost of $+£ 880$ as $£ 880 / 0.044=£ 20,000$ ).

- The mean cost of a stroke admission in the study price year of 2006 was $£ 2642$. Therefore the intervention is still cost-effective compared with control so long as there were less than $6204 / 2642=2.3$ more admissions per patient, on average, in the intervention arm compared with control over the three month period. (Note this is not total admissions, but 2.3 additional admissions compared with the control arm.)

- for the ICER to be below $£ 30,000$, intervention arm patients must have no more than a average of 2.5 admissions per patient over the three month period.

Additional file 1: Table A2.1. Baseline characteristics of patients included in analysis 4.

Additional file 2: Table A2.2. Baseline characteristics of patients included in analyses 5 and 6 .

\section{Acknowledgements \& Funding}

The trial was funded by UK National Health Service Research and Development Health Technology Assessment Programme (project reference 01/73/03). We would like to thank all the patients and their relatives who participated in the trial, the research fellows who were responsible for screening, recruitment, and the day-to-day running of the trial-A Dixit, $T$ Black, and P Johnson-and all other medical and nursing teams at all the hospitals involved.

The views and opinions expressed therein are those of the authors and do not necessarily reflect those of the Department of Health. 


\section{Author details}

${ }^{1}$ Faculty of Health, University of East Anglia, Norwich, UK. ${ }^{2}$ Stroke Research Group, Institute for Ageing and Health, Newcastle University, UK. ${ }^{3}$ Ageing \& Stroke Medicine, Department of Cardiovascular Sciences, University of Leicester, Leicester General Hospital, Leicester, UK. ${ }^{4}$ Institute for Ageing and Health, Newcastle University, Newcastle Upon Tyne, UK.

\section{Authors' contributions}

JFP was the principal investigator, developed the trial, sought and obtained funding. CJ oversaw the statistical analysis. AKM was the CHHIPS trial coordinator and responsible for data management. TGR \& GAF were coinvestigators responsible for developing the trial, applying for trial funding and were members of the trial steering committee. EW carried out the economic evaluation and drafted the manuscript. All authors read and reviewed manuscript drafts, and approved the final version.

\section{Competing interests}

The authors declare that they have no competing interests.

Received: 21 September 2009 Accepted: 23 March 2010 Published: 23 March 2010

\section{References}

1. Incidence of stroke in europe at the beginning of the 21st century. Stroke 2009, 40(5):1557-1563.

2. Carroll K, Murad S, Eliahoo J, Majeed A: Stroke incidence and risk factors in a population-based prospective cohort study. Office of National Statistics 2001

3. Martin J, Meltzer H, Elliot D: The prevalence of disability among adults. London: Office of Population Censuses and Surveys, HMSO 1988.

4. Comptroller and Auditor General: Reducing brain damage: faster access to better stroke care. London: National Audit Office 2005.

5. Ohwaki K, Yano E, Nagashima H, Hirata M, Nakagomi T, Tamura A: Blood pressure management in acute intracerebral hemorrhage: relationship between elevated blood pressure and hematoma enlargement. Stroke 2004, 35(6):1364-1367.

6. Ahmed N, Wahlgren G: High initial blood pressure after acute stroke is associated with poor functional outcome. J Intern Med 2001, 249(5):467-473.

7. Bhalla A, Wolfe CD, Rudd AG: The effect of $24 \mathrm{~h}$ blood pressure levels on early neurological recovery after stroke. J Intern Med 2001, 250(2):121-130.

8. Britton $\mathrm{M}$, Carlsson A: Very high blood pressure in acute stroke. J Intern Med 1990, 228(6):611-615.

9. Chamorro A, Vila N, Ascaso C, Elices E, Schonewille W, Blanc R: Blood pressure and functional recovery in acute ischemic stroke. Stroke 1998, 29(9):1850-1853.

10. Dandapani BK, Suzuki S, Kelley RE, Reyes-Iglesias Y, Duncan RC: Relation between blood pressure and outcome in intracerebral hemorrhage. Stroke 1995, 26(1):21-24.

11. Davalos A, Cendra E, Teruel J, Martinez M, Genis D: Deteriorating ischemic stroke: risk factors and prognosis. Neurology 1990, 40(12):1865-1869.

12. Dunne JW, Chakera T, Kermode S: Cerebellar haemorrhage-diagnosis and treatment: a study of 75 consecutive cases. Q J Med 1987 , 64(245):739-754

13. Qureshi Al, Safdar K, Weil J, Barch C, Bliwise DL, Colohan AR, Mackay B, Frankel MR: Predictors of early deterioration and mortality in black Americans with spontaneous intracerebral hemorrhage. Stroke 1995, 26(10):1764-1767.

14. Jorgensen HS, Nakayama H, Raaschou HO, Olsen TS: Effect of blood pressure and diabetes on stroke in progression. Lancet 1994, 344(8916):156-159.

15. Allen CM: Predicting outcome after acute stroke: role of computerised tomography. Lancet 1984, 2(8400):464-465.

16. Osaki $Y$, Matsubayashi $K$, Yamasaki M, Okumiya $K$, Yoshimura $K$ Hamashige N, Doi Y: Post-stroke hypertension correlates with neurologic recovery in patients with acute ischemic stroke. Hypertens Res 1998, 21(3):169-173

17. Lindley RI, Amayo EO, Marshall J, Sandercock PA, Dennis M, Warlow CP. Acute stroke treatment in UK hospitals: the Stroke Association survey of consultant opinion. J R Coll Physicians Lond 1995, 29(6):479-484.
18. Geeganage C, Bath P: Interventions for deliberately altering blood pressure in acute stroke. Cochrane Database of Systematic Reviews 2008, 4: Art No: CD000039.

19. Drummond MF, Sculpher MJ, Torrance GW, O'Brien BJ, Stoddart GL: Methods for the economic evaluation of health care programmes, 3 edn. Oxford: Oxford University Press 2005

20. Guide to the methods of technology appraisal. [http://www.nice.org.uk/ media/B52/A7/TAMethodsGuideUpdatedJune2008.pdf].

21. Potter J, Robinson T, Ford G, James $M$, Jenkins D, Mistri A, Bulpitt C, Drummond A, Jagger C, Knight J, et al: CHHIPS (Controlling Hypertension and Hypotension Immediately Post-Stroke) Pilot Trial: rationale and design. J Hypertens 2005, 23(3):649-655.

22. Potter JF, Robinson TG, Ford GA, Mistri A, James M, Chernova J, Jagger C: Controlling hypertension and hypotension immediately post-stroke (CHHIPS): a randomised, placebo-controlled, double-blind pilot trial. Lancet Neurol 2009, 8(1):48-56.

23. Potter J, Mistri A, Brodie F, Chernova J, Wilson E, Jagger C, James M, Ford G, Robinson T: Controlling hypertension and hypotension immediately post stroke (CHHIPS)-a randomised controlled trial. Health Technol Assess 2009, 13(9):iii.

24. Duncan PW, Lai SM, Keighley J: Defining post-stroke recovery: implications for design and interpretation of drug trials. Neuropharmacology 2000, 39(5):835-841.

25. National Schedule of Reference Costs 2005-06. [http://www.dh.gov.uk/en/ Publicationsandstatistics/Publications/PublicationsPolicyAndGuidance/ DH_062884].

26. British Medical Association, Royal Pharmaceutical Society of Great Britain British National Formulary 51. London 2006

27. Fenwick E, Claxton K, Sculpher M: Representing uncertainty: the role of cost-effectiveness acceptability curves. Health Econ 2001, 10(8):779-787.

28. Mclnnes G, Burke TA, Carides G: Cost-effectiveness of losartan-based therapy in patients with hypertension and left ventricular hypertrophy: a UK-based economic evaluation of the Losartan Intervention for Endpoint reduction in hypertension (LIFE) study. J Hum Hypertens 2006, 20(1):51-58.

29. Lundkvist J, Ekman M, Kartman B, Carlsson J, Jonsson L, Lithell H: The costeffectiveness of candesartan-based antihypertensive treatment for the prevention of nonfatal stroke: results from the Study on COgnition and Prognosis in the Elderly. J Hum Hypertens 2005, 19(7):569-576.

30. Lindgren $P$, Buxton M, Kahan T, Poulter NR, Dahlof B, Sever PS, Wedel H, Jonsson B: Cost-effectiveness of atorvastatin for the prevention of coronary and stroke events: an economic analysis of the AngloScandinavian Cardiac Outcomes Trial-lipid-lowering arm (ASCOT-LLA). Eur J Cardiovasc Prev Rehabil 2005, 12(1):29-36.

31. Lubeck DP, Hubert HB: Self-report was a viable method for obtaining health care utilization data in community-dwelling seniors. J Clin Epidemiol 2005, 58(3):286-290.

32. Raina P, Torrance-Rynard V, Wong M, Woodward C: Agreement between self-reported and routinely collected health-care utilization data among seniors. Health Serv Res 2002, 37(3):751-774

33. Bowen J, Yaste C: Effect of a stroke protocol on hospital costs of stroke patients. Neurology 1994, 44(10):1961-1964.

34. Diringer MN, Edwards DF, Mattson DT, Akins PT, Sheedy CW, Hsu CY, Dromerick AW: Predictors of acute hospital costs for treatment of ischemic stroke in an academic center. Stroke 1999, 30(4):724-728.

35. Flynn RW, MacWalter RS, Doney AS: The cost of cerebral ischaemia. Neuropharmacology 2008, 55(3):250-256

36. Claxton K: The irrelevance of inference: a decision-making approach to the stochastic evaluation of health care technologies. J Health Econ 1999, 18(3):341-364

doi:10.1186/1478-7547-8-3

Cite this article as: Wilson et al:: Controlling hypertension immediately post stroke: a cost utility analysis of a pilot randomised controlled trial. Cost Effectiveness and Resource Allocation 2010 8:3. 